\title{
Imaging observations of nighttime mid-latitude F-region field-aligned irregularities by an MU radar ultra-multi-channel system
}

\author{
S. Saito ${ }^{1, *}$, M. Yamamoto ${ }^{2}$, and H. Hashiguchi ${ }^{2}$ \\ ${ }^{1}$ National Institute of Information and Communications Technology, Nukui-kita 4-2-1, Koganei, Tokyo 184-8795, Japan \\ ${ }^{2}$ Research Institute for Sustainable Humanosphere, Kyoto University, Gokasho, Uji, Kyoto 611-0011, Japan \\ *now at: Electronic Navigation Research Institute, Jindaiji-higashi 7-42-23, Chofu, Tokyo 182-0012, Japan
}

Received: 7 November 2007 - Revised: 8 April 2008 - Accepted: 29 April 2008 - Published: 5 August 2008

\begin{abstract}
Mid-latitude F-region field-aligned irregularities (FAIs) were studied by using the middle-and-upper atmosphere (MU) radar ultra-multi-channel system with the radar imaging technique. On 12 June 2006, F-region FAI echoes with a period of about one hour were observed intermittently. These echoes were found to be embedded in mediumscale traveling ionospheric disturbances (MSTIDs) observed as variations of total electron content (TEC). The echoes drifting away from (toward) the radar were observed in the depletion (enhancement) phase of the MSTID. The Doppler velocity of the echoes is consistent with the range rates in the the range-time-intensity (RTI) maps. Fine scale structures with a spatial scale of $10 \mathrm{~km}$ or less were found by the radar imaging analysis. Those structures with positive Doppler velocities (moving away from the radar) appeared to drift north(up-) westward, and those with negative Doppler velocities south- (down-) eastward approximately along the wavefronts of the MSTID. FAIs with positive Doppler velocities filling TEC depletion regions were observed.
\end{abstract}

Keywords. Ionosphere (Ionospheric irregularities; Midlatitude ionosphere; Plasma waves and instabilities)

\section{Introduction}

In the mid-latitude ionosphere, several kinds of plasma irregularities are known to exist in both the E- and F-regions. Nighttime F-region 3-m-scale field-aligned plasma irregularities (FAIs) associated with the mid-latitude spread-F were first reported by Fukao et al. (1988). Compared with those in the E-region, such as quasi-periodic (QP) echoes associ-

Correspondence to: S. Saito

(susaito@enri.go.jp) ated with the sporadic-E $\left(E_{s}\right)$ layer (Yamamoto et al., 1991), they have not been studied well. Only a few studies in Japan (Fukao et al., 1988, 1991; Saito et al., 1998, 2002) and in Puerto Rico (Swartz et al., 2000) using radars have reported the mid-latitude F-region FAIs. This is partly because the echoes associated with the mid-latitude F-region FAIs are relatively weak and a powerful radar is required to observe them.

Several characteristics have been revealed by the studies sited above. Nighttime mid-latitude F-region FAIs associated with medium-scale traveling ionospheric disturbances (MSTIDs) with typical wavelengths of $100-500 \mathrm{~km}$ have been observed (Saito et al., 1998, 2002). However, F-region FAIs are less frequently observed than MSTIDs. Accordingly, the mechanism of generating 3-m-scale FAIs in the Fregion has not been understood well.

Perkins (1973) has proposed that the ionosphere can be unstable and the wave structure excited by the instability, called the Perkins instability, preferentially propagates southwestward or northeastward (see also Zhou and Mathews, 2006). Nighttime mid-latitude MSTIDs are often explained by the Perkins instability. However, it is also known that the linear growth rate of the instability is too small to explain the MSTID phenomenon. The Perkins instability cannot explain how 3-m-scale FAIs are generated, because they are supposed to be secondary waves generated after non-linear evolution of primary waves.

Kelley et al. (1991) proposed that interchange-type instability could be excited by the electron density gradient associated with MSTIDs and the neutral wind. In this case, a certain slope of MSTIDs with $\nabla n_{e} \cdot \mathbf{u}<0$, where $n_{e}$ and $\mathbf{u}$ are the electron density and the neutral wind velocity, respectively, would be unstable, and the other slope would be stable. Using the middle-and-upper (MU) atmosphere radar (Fukao et

Published by Copernicus Publications on behalf of the European Geosciences Union. 


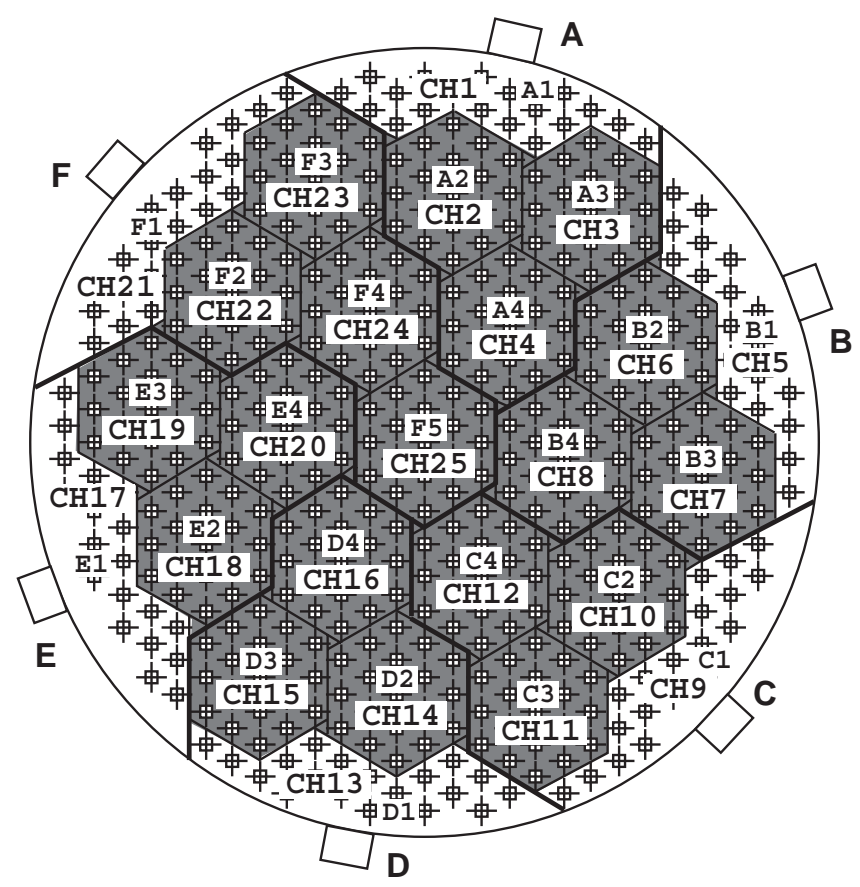

Fig. 1. Layout of the MU radar antenna sub-arrays. The 19 shaded sub-arrays were used to take data independently for imaging analysis. All 25 sub-arrays were used for transmission.

al., 1985a, b) multi-beam experiment, Saito et al. (2002) reported that echoes tend to appear at the northern slope of MSTIDs. However, it was difficult to see the precise relationship between the MSTIDs and FAIs, nor fine scale structures of FAIs, as their observations were conducted without interferometry.

Zhou et al. (2005) showed by numerical simulation that the non-linear Perkins instability would have a larger growth rate and may generate $10-\mathrm{km}$-scale irregularities. They also showed that the interchange-type instability can generate small-scale irregularities.

The radar imaging technique is now widely used for studying fine structures of ionospheric irregularities from equatorial to high latitudes (Hysell, 1996; Hysell and Burcham, 2000; Hysell and Chau, 2002; Hysell et al., 2002, 2004; Bahcivan et al., 2005; Saito et al., 2006, 2007). With this technique, structures smaller than the radar beam width can be resolved. Using the MU radar ultra-multi-channel system, we conducted multi-beam radar-imaging experiment to study the structures of mid-latitude F-region FAIs in detail.

\section{Experiment}

All 25 antenna sub-arrays of the MU radar ultra-multichannel system were used for transmission, and 19 subarrays with a regular hexagonal shape recorded returned signals independently (Fig. 1). The observations were con-

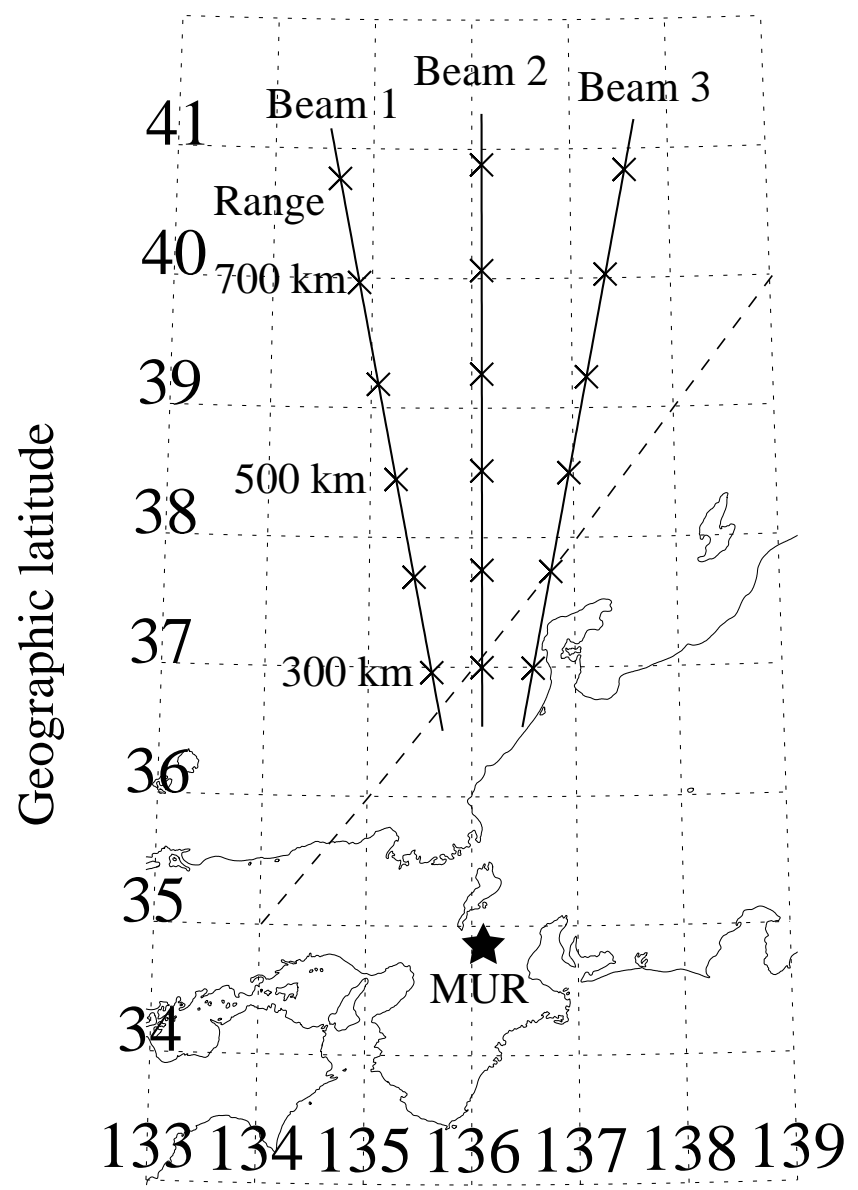

\section{Geographic longitude}

Fig. 2. Geometry of the observation. Three thick lines show the center of the radar beam with crosses marked at every $100 \mathrm{~km}$ range.

ducted from 20:00 to 04:00 JST (UT+9h) on 12-15 June and 11-13 July 2006. E- and F-regions were observed alternately. Parameters of the MU radar observations of the F-region are summarized in Table 1. Figure 2 shows the geometry of the experiment. The beams used for the observations were named beams 1,2, and 3 from west to east, respectively. Due to the high aspect angle sensitivity of FAI echoes, echoes can be observed only when the radar k-vector is perpendicular to the geomagnetic field line. The perpendicularity condition in the $-3 \mathrm{~dB}$ beam width $\left(4.5^{\circ}\right)$ was achieved at altitudes $208-336 \mathrm{~km}, 224-347 \mathrm{~km}$, and $161-295 \mathrm{~km}$ for beams 1,2 , and 3 , respectively. These altitudes correspond to ranges $350-619 \mathrm{~km}, 384-648 \mathrm{~km}$, and $274-547 \mathrm{~km}$, respectively. Although the MU radar can switch beam directions on a pulse-to-pulse basis, beam directions in this experiment were switched after completion of a measurement for each beam in order to keep their Doppler spectrum widths wide enough. One experiment cycle took $91 \mathrm{~s}$, and nominal dwell time of each F-region beam was about $17 \mathrm{~s}$. 
Table 1. Parameters of the MU radar observations.

\begin{tabular}{ll}
\hline Number of beams & 3 \\
Beam direction (Azimuth, Zenith) & $\left(-10.43^{\circ}, 57.19^{\circ}\right),\left(0.00^{\circ}, 57.84^{\circ}\right),\left(10.43^{\circ}, 57.19^{\circ}\right)$ \\
Sensitive altitude range & $208-336 \mathrm{~km}, 224-347 \mathrm{~km}, 161-295 \mathrm{~km}$ \\
Pulse code & 11 -bit Barker code \\
Sub-pulse length & $32 \mu \mathrm{s}(4.8 \mathrm{~km})$ \\
Inter pulse period & $8 \mathrm{~ms}$ \\
Doppler velocity bandwidth & $\pm 201 \mathrm{~m} \mathrm{~s}^{-1}$ \\
Number of incoherent integration & 5 \\
Peak power & $1 \mathrm{MW}$ \\
\hline
\end{tabular}

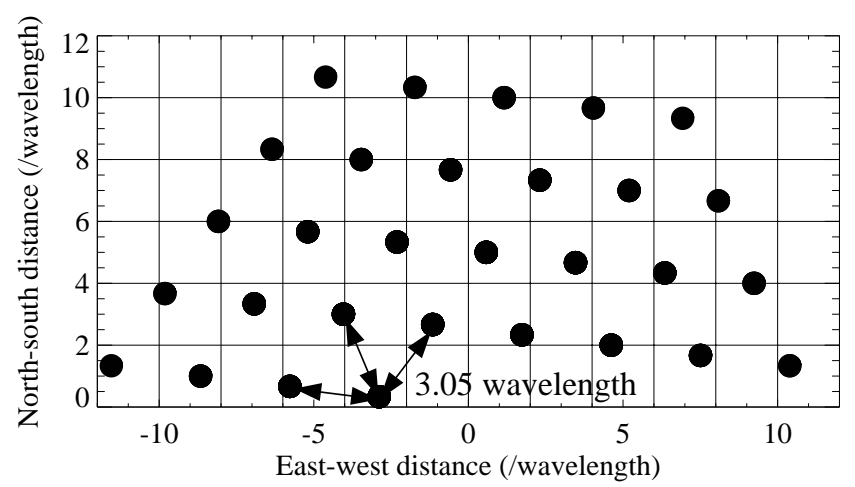

Fig. 3. Non-redundant baselines achieved by the 19 antenna subarrays and used for imaging analysis.

\section{Imaging analysis}

The analysis procedures of radar echoes are basically the same as used for the E-region measurements by Saito et al. (2006). Complex Doppler spectra were calculated by fast Fourier transform (FFT). The two-dimensional visibility function was estimated for 30 non-redundant baselines achieved by the 19 antenna sub-arrays (Fig. 3). The coherence functions were divided into four segments in the Doppler velocity domain. Images of echoes were reconstructed by the maximum entropy method (Hysell, 1996) for three of the four segments of Doppler velocities, toward the radar $\left(-151\right.$ to $\left.-50 \mathrm{~m} \mathrm{~s}^{-1}\right)$, around zero $\left(-50\right.$ to $\left.+50 \mathrm{~m} \mathrm{~s}^{-1}\right)$, and away $\left(+50\right.$ to $\left.+151 \mathrm{~m} \mathrm{~s}^{-1}\right)$ from the radar. The fastest segment $\left(-151\right.$ to $-201 \mathrm{~m} \mathrm{~s}^{-1}$ and +151 to $\left.+201 \mathrm{~m} \mathrm{~s}^{-1}\right)$, which had more chance to be frequency aliased than the other segments, was not used, because the F-region echo spectra are broad with relatively small Doppler shifts that are lower than the ion acoustic velocity (about 300 to $400 \mathrm{~m} \mathrm{~s}^{-1}$ ). In the present study, the positive and negative Doppler velocities refer to the motion away from and toward the radar, respectively.
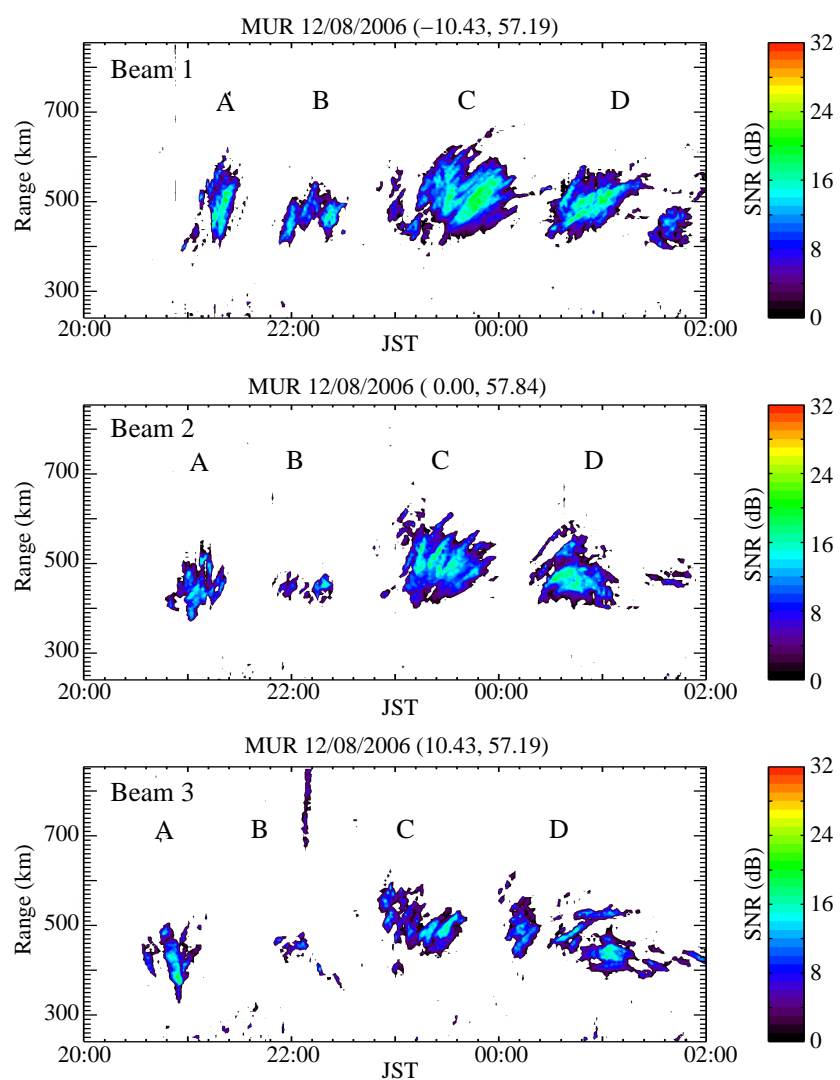

Fig. 4. Echo power observed on 12 June 2006 in the RTI format $(\mathrm{JST}=\mathrm{UT}+9 \mathrm{~h})$. Upper, middle, and lower panel show the data from beams 1,2 , and 3 , respectively.

\section{Results}

Figure 4 shows the echo strength observed by the three beams on 12 July 2006 in the range-time-intensity (RTI) format. Echoes were observed at the expected ranges in all beams. Echoes with signal-to-noise ratio (SNR) as strong as $15 \mathrm{~dB}$ were intermittently observed around 21:00, 22:00, 23:00, and 01:00 JST (UT+9h) with duration of 0.5 to $1 \mathrm{~h}$ as marked as A to D, respectively, in the figure. Most of echoes were observed with positive range rates, i.e. the range of the 

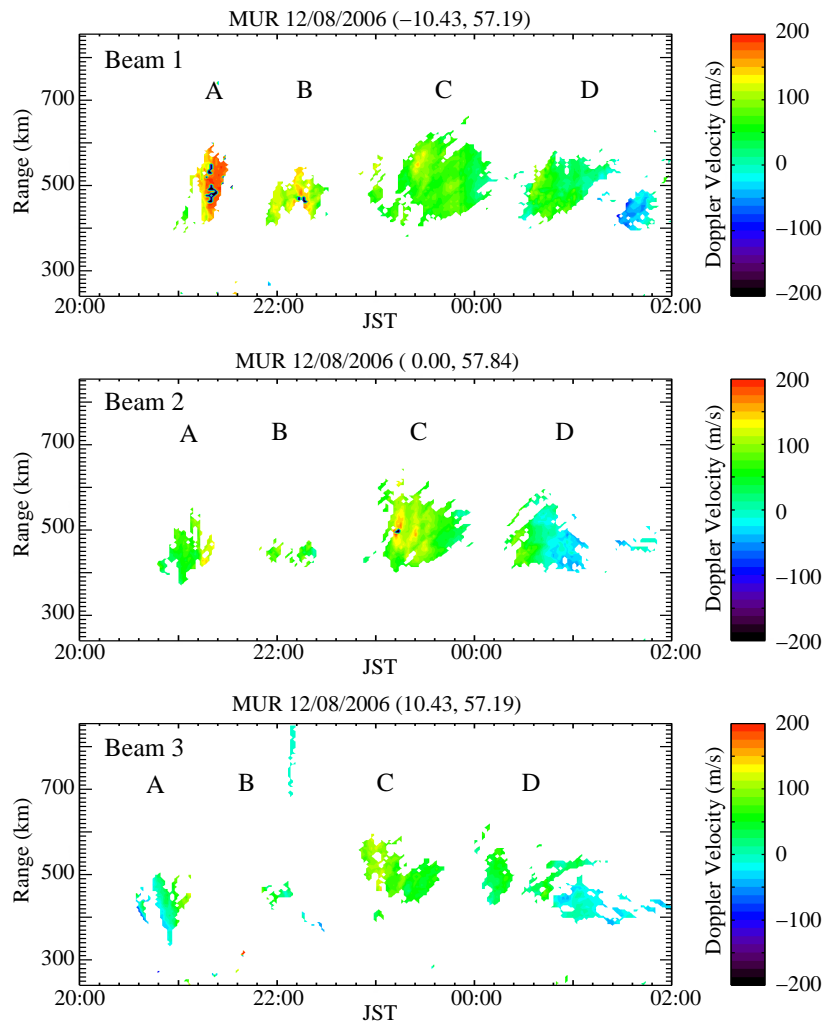

Fig. 5. Same as Fig. 4, except the Doppler velocity is shown.

echo pattern increased with time. Echo cluster A in beam 3 and echo cluster $\mathrm{D}$ in all beams have both positive and negative range rates. The first half of echo cluster $\mathrm{D}$ has positive range rates, and the latter half of the echoes has negative range rates.

Figure 5 shows the Doppler velocities observed by the three beams over the same period. Doppler velocities of echoes with SNR larger than $0 \mathrm{~dB}$ are plotted. The Doppler velocities were mostly positive, and about 50 to $150 \mathrm{~m} \mathrm{~s}^{-1}$. However, some echoes have negative Doppler velocities of about $-50 \mathrm{~m} \mathrm{~s}^{-1}$. There are several echoes with very high negative Doppler velocities as fast as $-200 \mathrm{~m} \mathrm{~s}^{-1}$ in the echo clusters $\mathrm{A}$ and $\mathrm{B}$ in beam 1 and in the echo cluster $\mathrm{C}$ in beam 2. However, spectral analysis of these echoes shows that they are most likely due to frequency aliasing by very fast, positive Doppler velocity exceeding the Nyquist limit $\left(202 \mathrm{~m} \mathrm{~s}^{-1}\right)$. Echoes with positive range rates have positive Doppler velocities (moving away from the radar) and those with negative range rates had negative ones (moving toward the radar). The observed Doppler velocities were consistent with the range rates, as reported in previous works (e.g. Fukao et al., 1991).

Figure 6 shows an example of a sequence of radar images, when a strong echo cluster (marked as C in Fig. 4) was observed. Images for each one of the Doppler velocity segments ( -150 to $-50 \mathrm{~m} \mathrm{~s}^{-1},-50$ to $+50 \mathrm{~m} \mathrm{~s}^{-1}$, and +50 to
$+150 \mathrm{~m} \mathrm{~s}^{-1}$ ) were merged by assigning weights proportional to echo power and image intensity. The colors blue, green, or red, was assigned to each Doppler velocity segment: toward, zero, or away, respectively. Each color represents a range from 0 to $3 \mathrm{~dB}$ with six color steps to show weak echoes more clearly. The images were projected on a horizontal plane at an altitude of $300 \mathrm{~km}$. Echo cluster $\mathrm{C}$ contains a few echoes with Doppler velocities higher than $150 \mathrm{~m} \mathrm{~s}^{-1}$. These echo spectra are broad, and a significant amount of echo power in the spectral ranges that were used for imaging analysis $\left(-150\right.$ to $\left.+150 \mathrm{~m} \mathrm{~s}^{-1}\right)$. Thus, the images of such echoes with very high Doppler velocities were also successfully reconstructed. The images shown in Fig. 6 are dominated by a red color, indicating that the Doppler velocity was higher than $50 \mathrm{~m} \mathrm{~s}^{-1}$. The Doppler echoing regions consists of substructures with scale sizes less than $10 \mathrm{~km}$. As indicated by white circles in each panel, these substructures appeared to drift north- (up-) westward. This is consistent with Swartz et al. (2000)'s finding of 1 to $100 \mathrm{~km}$ scale layering in the mid-latitude F-region irregularities. After 00:40 JST on 13 June 2006, echoes with negative range rates were observed in beam 2 in the latter half of the echo cluster D in Fig. 4. Figure 7 shows a sequence of radar images of the echoes from the latter half of the cluster D in beam 2. All echoes in this echo cluster had Doppler velocities between -150 and $+150 \mathrm{~m} \mathrm{~s}^{-1}$. The images were dominated by a green color, indicating that the Doppler velocity was mostly negative (toward) and slower than $-50 \mathrm{~m} \mathrm{~s}^{-1}$. Echoing regions also consist of patches with scale sizes less than $10 \mathrm{~km}$. These echoing regions appeared to drift south (down) eastward. Similar images were also observed in the echo clusters A and B.

From 20:00 JST on 12 July 2006 to $02: 00 \mathrm{JST}$ on 13 July 2006, in the region where MU radar echoes were observed, strong medium-scale traveling ionospheric disturbances (MSTIDs) were observed in the total electron contents (TECs) measured by the GEONET GPS receiver network. MSTIDs were observed as TEC perturbation which was derived every $30 \mathrm{~s}$ as deviation from one hour moving average of TECs. In converting slant TECs to vertical ones, the contribution of electrons to the slant TECs is assumed to be entirely from a virtual thin layer at an altitude of $300 \mathrm{~km}$. This is the same method as that developed by Saito et al. (1998). Figure 8 shows the temporal variations of TEC perturbation along the line passing through the region observed by the MU radar which is drawn in Fig. 2 as an oblique dashed line. Oblique stripes from top left to bottom right indicate the occurrence of MSTIDs propagating southwestward. The amplitude was about \pm 1 TECU $\left(10^{16} \mathrm{~m}^{-2}\right)$. The echoes with positive (negative) range rates were observed in the negative (positive) phase of MSTIDs. Figure 9 shows the relationship between FAI images and MSTIDs. The FAI images are projected on a horizontal plane at an altitude of $300 \mathrm{~km}$. All these echoes have positive Doppler velocity (moving away from the radar) as indicated by reddish colors. It can be seen that the FAIs filled the TEC depletion region, 


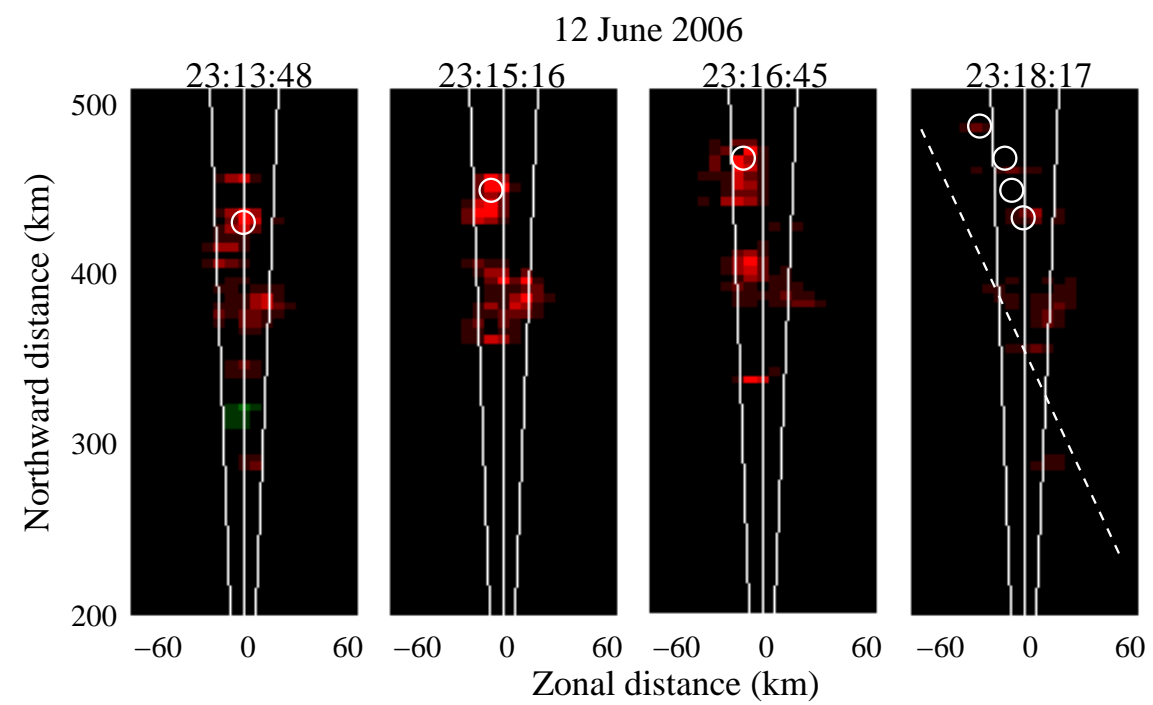

Fig. 6. Sequential images of echoes from beam 2 cluster $C$ from 23:13:48 to 23:18:17 JST on 12 June 2006. The images are projected on a horizontal plane at an altitude of $300 \mathrm{~km}$ along the geomagnetic field line. White circles in the rightmost panel show the approximate positions of echo patches in these four images as also indicated by circles in other panels. The dashed line in the rightmost panel indicates the wavefront direction of the MSTID (at an arbitrary phase).

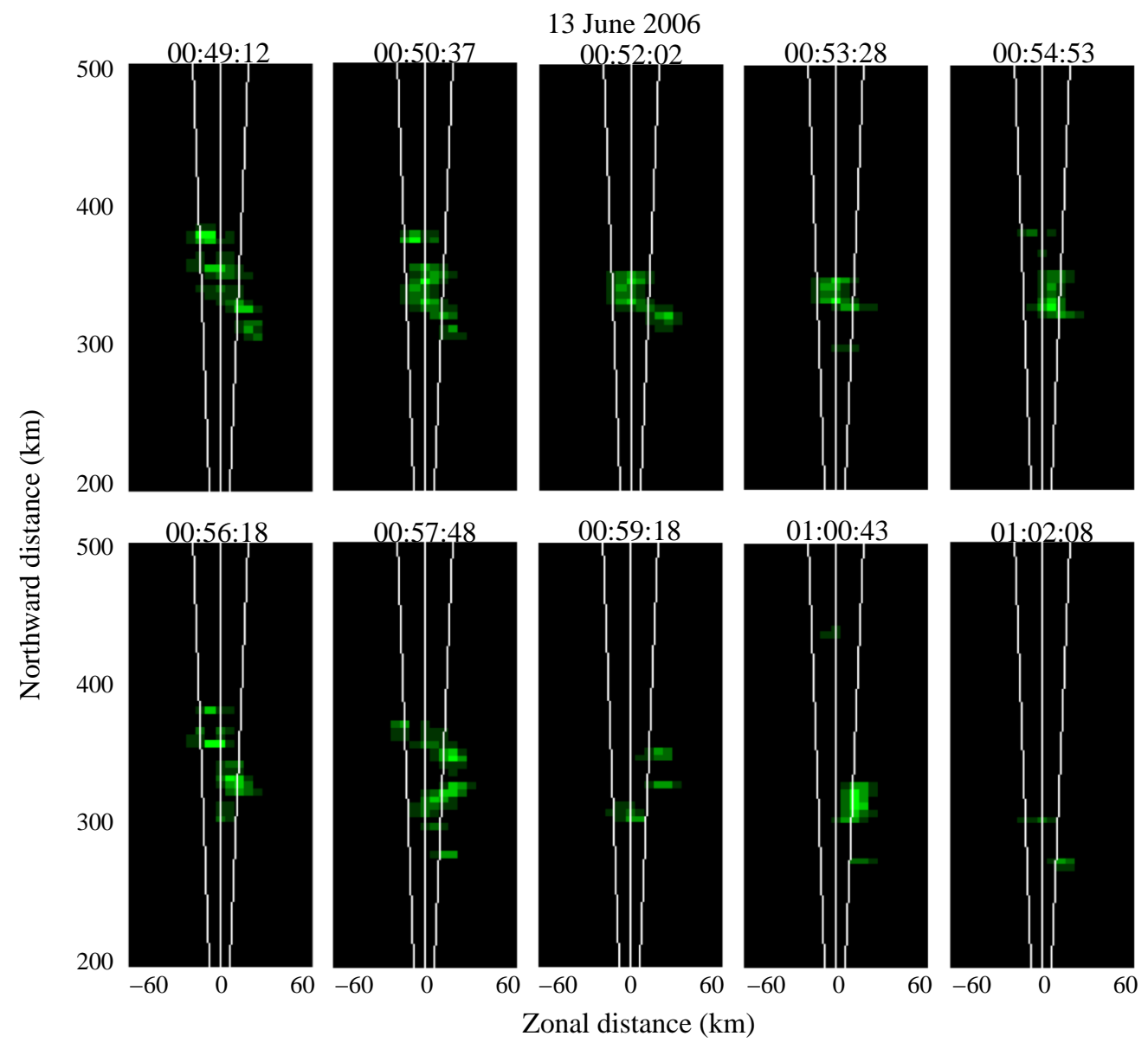

Fig. 7. Same as Fig. 6, except echoes from beam 2 cluster D from 00:49:12 to 01:02:08 JST on 13 June 2006 are shown. 


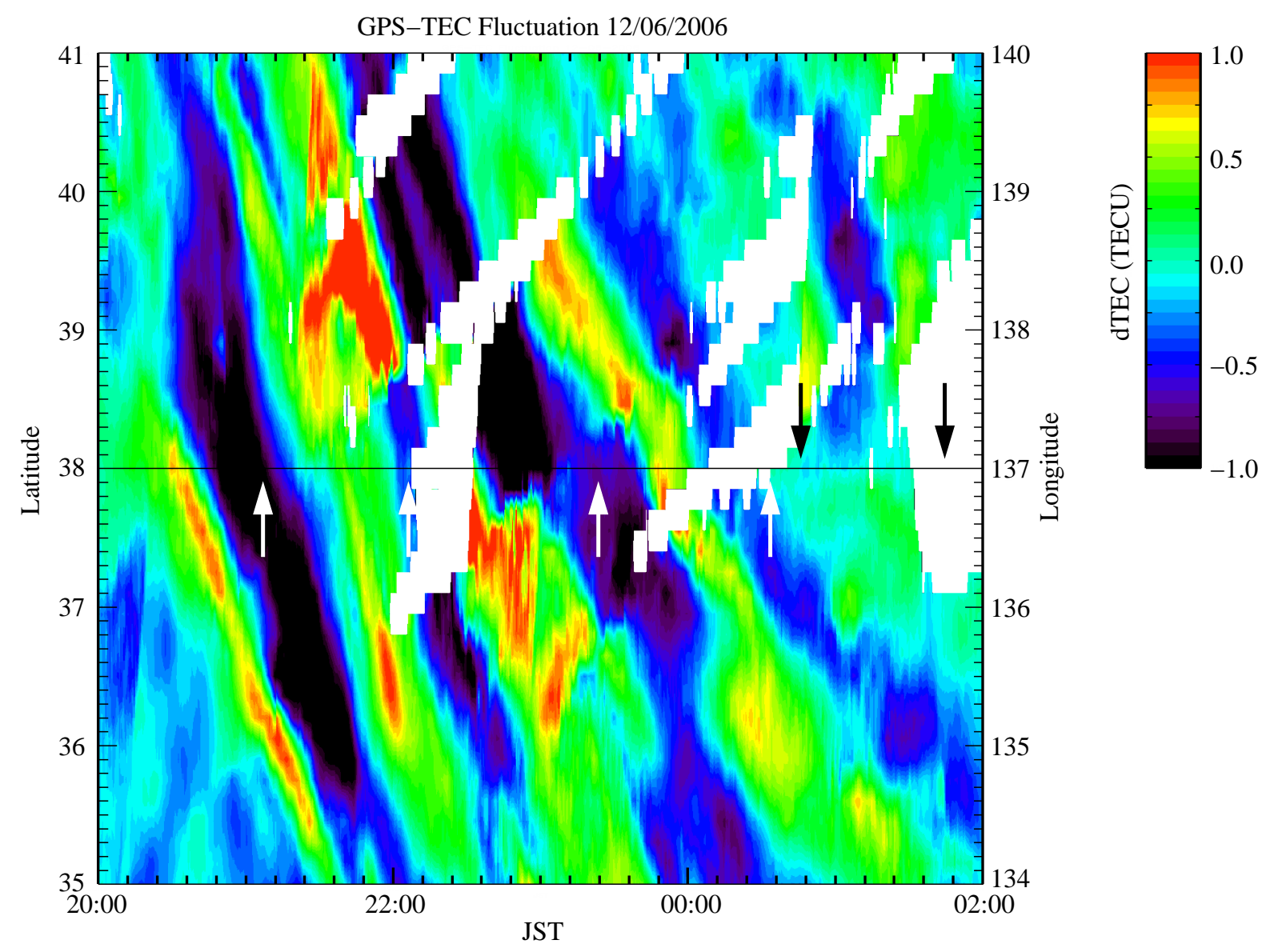

Fig. 8. TEC perturbation along the line from $\left(35^{\circ} \mathrm{N}, 134^{\circ} \mathrm{E}\right)$ to $\left(41^{\circ} \mathrm{N}, 140^{\circ} \mathrm{E}\right)$ (dashed line in Fig. 2 approximately perpendicular to the MSTID wavefront). Up and down arrows indicate the times when FAIs with positive and negative range rates, respectively, appeared in beam 2 .

and moved southwestward as a whole clearly together with the MSTID.

\section{Discussion and summary}

Our observations by the MU radar ultra-multi-channel system show that substructures $(\sim 10 \mathrm{~km}$-scale $)$ of FAI echoes drifted approximately along the MSTID wave front, while echoing regions as a whole drifted southwestward together with the MSTID. These 10-km-scale FAI substructures could be related to the kilometer-scale layered structure reported by Mathews et al. (2001). The drift of these echo patches may be explained by the electric field associated with the MSTID. Figure 10 illustrates the motion of the irregularity patches and its relation to the MSTID. At night in the Northern Hemisphere, the neutral wind velocity in the F-region is generally southeastward. The wind, $\boldsymbol{U}$, generates the Pedersen current, $\boldsymbol{J}=\sigma_{P} \boldsymbol{U} \times \boldsymbol{B}$, where $\sigma_{P}$ is the Pedersen conductivity, as shown in Fig. 10. According to Saito et al. (2002), in the TEC depletion region $\left(d N_{e}<0\right)$, the polarization electric field, $E_{p}$, would be northeastward and lift up the ionosphere. On the other hand, in the TEC enhancement region $\left(d N_{e}>0\right)$, the polarization electric field, $E_{p}$, would be southwestward. By the $E_{p}$ associated with the MSTID and the geomagnetic field, $\boldsymbol{E}_{p} \times \boldsymbol{B}$ drift would be north- (up-) westward (south- (down) eastward) in the TEC depletion (enhancement) region (see also Otsuka et al., 2007). Using satellite observations and a $630 \mathrm{~nm}$ airglow imager, Shiokawa et al. (2003) found that the plasma drift was along the MSTID wavefront and was northwestward (southeastward) in the $630 \mathrm{~nm}$ airglow depletion (enhancement) region. This corresponds to the ionosphere that is uplifted (pushed down), and hence the TEC depletion (enhancement) region, and is consistent with our finding of the small-scale echo patches drift. The small-scale patches seem to move with the background plasma. This agrees with the numerical simulation results by Zhou et al. (2006). For quantitative verification if the FAI patches really move with background plasma, neutral wind measurements are necessary. The Fabry-Perot interferometer of Nagoya University at the MU radar site was unfortunately not in operation on 12 


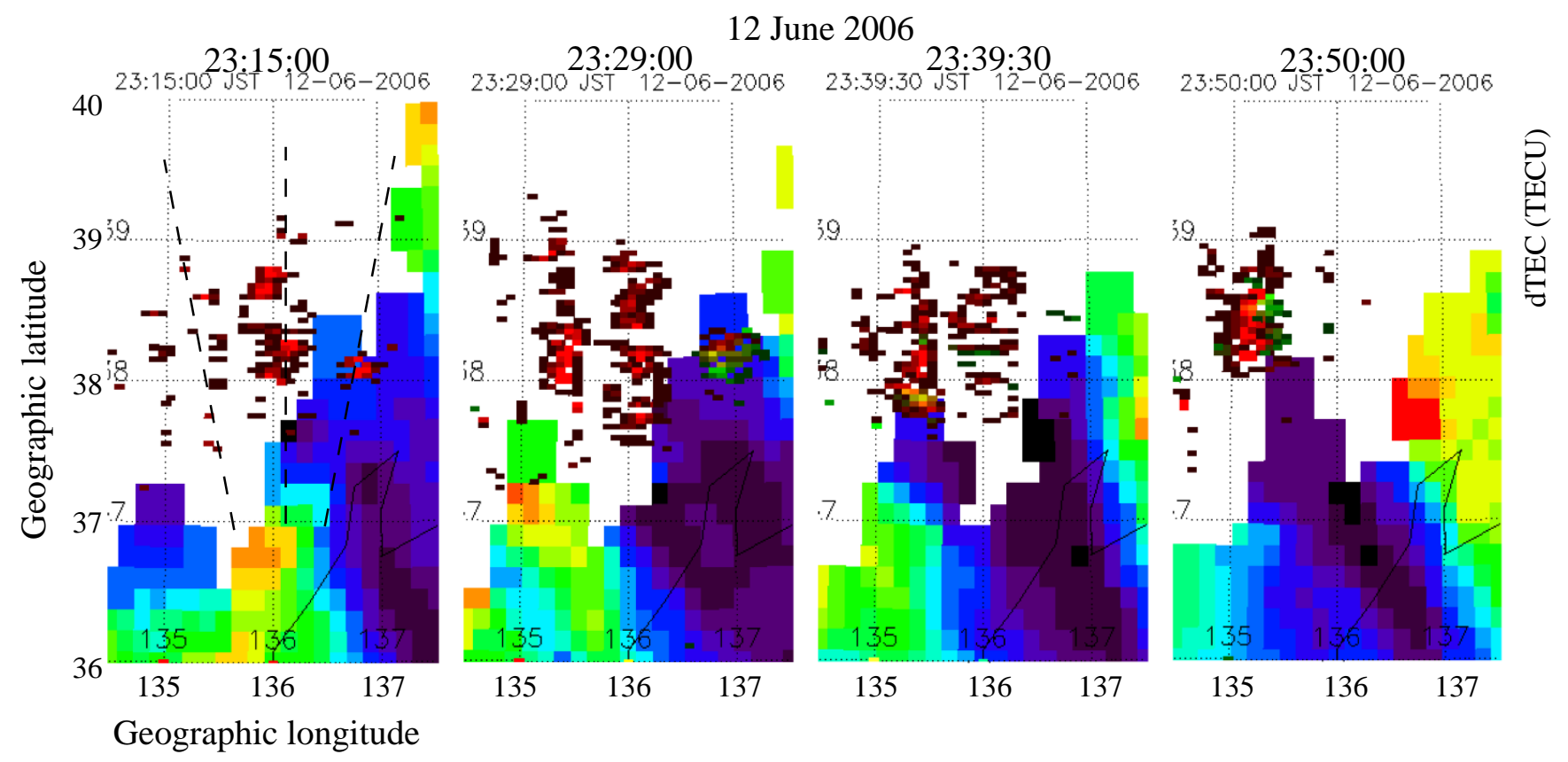

Fig. 9. Sequential images of echoes from beam 1, 2, and 3 together with TEC perturbation observed from 23:15 to 23:50 JST on 12 July 2006. The images are projected on a horizontal plane at an altitude of $300 \mathrm{~km}$ along the geomagnetic field line. Dashed lines in the images show the center of the radar beams.

June 2006 due to maintenance. In our future studies, simultaneous observations of FAI images and the neutral wind are planned.

Recently, Saito et al. (2007) reported that MSTIDs are closely related to quasi-periodic (QP) echoes in the E-region as evidence of the E- and F-region coupling. They have also pointed out different scale sizes in the E- and F-regions: 100 to $500 \mathrm{~km}$ for MSTIDs and several kilometers for QP echoes. Existing theories regarding E- and F-region coupling, which assume the same scale sizes in both regions, would have difficulty in explaining the difference in scale sizes. In this experiment, we found smaller scale $(\sim 10 \mathrm{~km})$ structures in the F-region FAIs associated with MSTIDs. In fact, on 12 June 2006, E-region echoes, that could be classified as QP echoes, were observed. Although MSTIDs do not always accompany FAIs, our results show possibility that small-scale structures exists in the F-region with a scale size comparable to that of the E-region.

We showed that the FAIs were found filling the TEC depletion region. Using an MU radar multi-beam experiment without interferometry, Saito et al. (2002) reported that echoes tend to appear at the northern slope of MSTIDs. However, our experiment with better spatial resolution showed that these echoes with positive range rate were distributed over the TEC depletion region of the MSTID irrespective of TEC gradients. This situation is quite similar to the case of plasma bubbles. The western wall of a plasma bubble is more unstable with the interchange instability due to the eastward thermospheric neutral wind. However, 3-m-scale FAIs fill entire

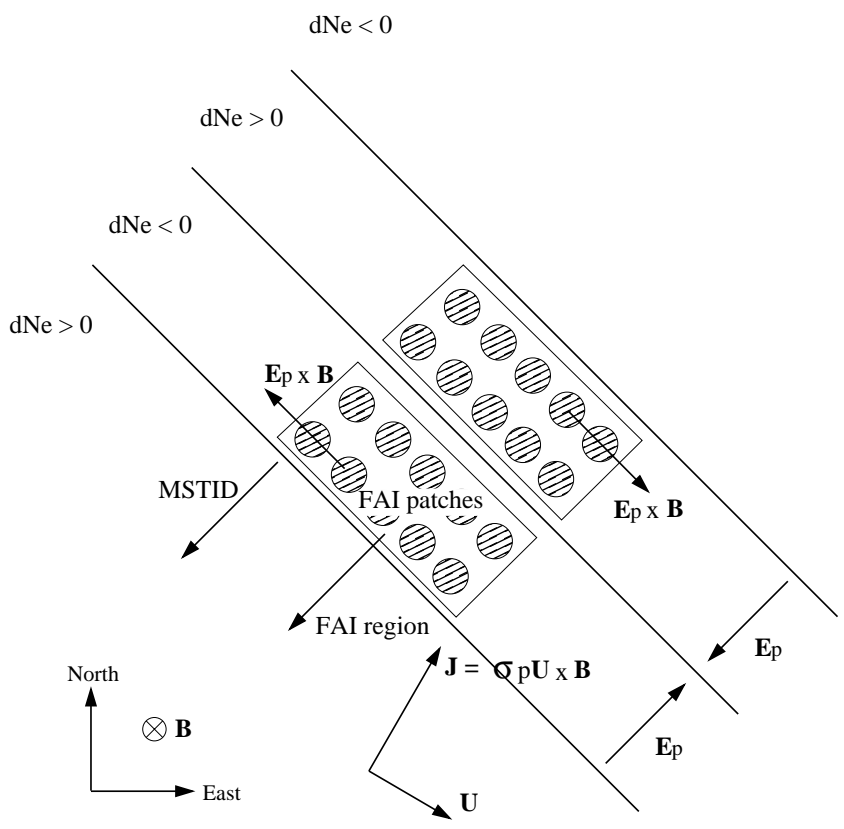

Fig. 10. Schematic illustration of motion of FAI patches with MSTIDs.

plasma bubbles from the western to the eastern walls (Otsuka et al., 2004). In the case of MSTIDs, even though FAIs could be generated at a certain phase of MSTIDs, they could soon develop into other regions. To test this hypothesis, we need to trace the development of FAIs. Multi-beam imaging 
observations can distinguish whether they are generated there or generated far away and drifted into the radar field-of-view. Unfortunately, no FAIs that were observed in our experiment were generated in the radar field-of-view. Even the echo cluster $\mathrm{D}$, which may look generated in the radar field-of-view if we look at the RTI map of only one beam, was in fact generated outside the radar field-of-view. It is necessary to conduct more experiments of this kind to enhance the chance to encounter "fresh" F-region FAIs.

Acknowledgements. The MU radar belongs to and is operated by the Research Institute for Sustainable Humanosphere (RISH) of Kyoto University. The GEONET GPS receiver network is operated by Geographical Survey Institute (GSI), Japan. TEC data are provided by Kyoto University.

Topical Editor U.-P. Hoppe thanks J. D. Mathews and another anonymous referee for their help in evaluating this paper.

\section{References}

Bahcivan, H., Hysell, D. L., Larsen, M. F., and Pfaff, R. F: The $30 \mathrm{MHz}$ imaging radar observations of auroral irregularities during the JOULE campaign, J. Geophys. Res., 110, A05307, doi:10.1029/2004JA010975, 2005.

Fukao, S., Sato, T., Tsuda, T., Kato, S., Wakasugi, K., and Makihara, T.: The MU radar with an active phased array system 1. Antenna and power amplifiers, Radio Sci., 20, 1155-1168, 1985a.

Fukao, S., Tsuda, T., Sato, T., Kato, S., Wakasugi, K., and Makihara, T.: The MU radar with an active phased array system 1. In-house equipment, Radio Sci., 20, 1169-1176, 1985 b.

Fukao, S., McClure, J. P., Ito, A., Sato, T., Kimura, I., Tsuda, T., and Kato, S.: First VHF radar observation of midlatitude Fregion field-aligned irregularities, Geophys. Res. Lett., 8, 768$771,1988$.

Fukao, S., Kelley, M. C., Shirakawa, T., Takami, T., Yamamoto, Y., Tsuda, T., and Kato, S.: Turbulent upwelling of the mid-latitude ionosphere 1., Observational results by the MU radar, J. Geophys. Res., 96, 3725-3746, 1991.

Hysell, D. L.: Radar imaging of equatorial F region irregularities with maximum entropy interferometry, Radio Sci., 31, 15671578, 1996.

Hysell, D. L. and Burcham, J. D.: The 30-MHz radar interferometer studies of midlatitude E region irregularities, J. Geophys. Res., 105, 12 797-12 812, 2000.

Hysell, D. L. and Chau, J. L.: Imaging radar observations and nonlocal theory of large-scale plasma waves in the equatorial electrojet, Ann. Geophys., 20, 1167-1179, 2002, http://www.ann-geophys.net/20/1167/2002/.

Hysell, D. L., Yamamoto, M., and Fukao, S.: Imaging radar observations and theory of type I and type II quasi-periodic echoes, J. Geophys. Res., 107(A11), 1360, doi:10.1029/2002JA009292, 2002.

Hysell, D. L., Larsen, M. F., and Zhou, Q. H.: Common volume coherent and incoherent scatter observations of mid-latitude sporadic E-layers and QP echoes, Ann. Geophys., 22, 3277-3290, 2004, http://www.ann-geophys.net/22/3277/2004/.

Kelley, M. C., S. Fukao, S., Tsuda, T., and Kato, S.: Turbulent upwelling of the mid-latitude ionosphere 2., Theoretical framework, J. Geophys. Res., 96, 3747-3753, 1991.
Mathews, J. D., González, S., Sulzer, M. P., Zhou, Q. H., Urbina, J., Kudeki, E., and Franke, S.: Kilometer-scale layered structures inside spread-F, Geophys. Res. Lett., 28, 4167-4170, 2001.

Otsuka, Y., Shiokawa, K., Ogawa, T., Yokoyama, T., Yamamoto, M., and Fukao, S.: Spatial relationship of equatorial plasma bubbles and field-aligned irregularities observed with an all-sky airglow imager and the Equatorial Atmosphere Radar, Goephys. Res. Lett., 24, L20802, doi:10.1029/2004GL020869, 2004.

Otsuka, Y., Onoma, F., Shiokawa, K., Ogawa, T., Yamamoto, M., and Fukao, S.: Simultaneous observations of nighttime medium-scale traveling ionospheric disturbances and E-region field-aligned irregularities at midlatitude, J. Geophys. Res., 112, A06317, doi:10.1029/2005JA011548, 2007.

Perkins, F.: Spread F and ionospheric currents, J. Geophys. Res., 78, 218-226, 1973.

Saito, A., Fukao, S., and Miyazaki, S.: High resolution mapping of TEC perturbations with the GSI GPS network over Japan, Geophys. Res. Lett., 25, 3079-3082, 1998.

Saito, A., Nishimura, M., Yamamoto, M., Fukao, S., Tsugawa, T., Otsuka, Y., Miyazaki, S., and Kelley, M. C.: Observations of traveling ionospheric disturbances and 3-m scale irregularities in the nighttime F-region ionosphere with the MU radar and a GPS network, Earth Planets Space, 54, 31-44, 2002.

Saito, S., Yamamoto, M., Hashiguchi, H., and Maegawa, A.:, Observation of three-dimensional structures of quasi-periodic echoes associated with mid-latitude sporadic-E layers by MU radar ultra-multi-channel system, Geophys. Res. Lett., 33, L14109, doi:10.1029/2005GL025526, 2006.

Saito, S., Yamamoto, M., Hashiguchi, H., and Maegawa, A.:, Observational evidence of coupling between quasi-periodic echoes and medium scale traveling ionospheric disturbances, Ann. Geophys., 25, 2185-2194, 2007,

http://www.ann-geophys.net/25/2185/2007/.

Shiokawa, K., Otsuka, Y., Ihara, C., Ogawa, T., and Rich, F. J.: Ground and satellite observations of nighttime medium-scale traveling ionospheric disturbance at midlatitude, J. Geophys. Res., 108, SIA 3-1, doi:10.1029/2002JA009639, 2003.

Swartz, W. E., Kelley, M. C., Makela, J. J., Collins, S. C., Kudeki, E., Franke, E. S., Urbina, J., Aponte, N., Sulzer, M. P., and González, S. A.: Coherent and incoherent scatter radar observations during intense mid-latitude spread F, Geophys. Res. Lett., 27, 2829-2832, 2000.

Yamamoto, M., Fukao, S., Woodman, R. F., Ogawa, T., Tsuda, T., and Kato, S.: Mid-latitude E-region field-aligned irregularities observed with the MU radar, J. Geophys. Res., 96, 15943$15949,1991$.

Zhou, Q., Mathews, J. D., Du, Q., and Miller, C. A.: A preliminary investigation of the pseudo-spectral method numerical solution of the Perkins instability equations in the homogeneous TEC case, J. Atmos. Solar-Terr. Phys., 67, 325-335, 2005.

Zhou, Q. and Mathews, J. D.: On the physical explanation of the Perkins instability, J. Geophys. Res., 111, A12309, doi:10.1029/2006JA011696, 2006.

Zhou, Q., Mathews, J. D., Miller, C. A., and Seker, I.: The evolution of nighttime mid-latitude mesoscale F-region structures: A case study utilizing numerical solution of the Perkins instability equations, Planet. Space Sci., 54, 710-718, 2006. 